\title{
Micro ATR FTIR imaging of hanging drop protein crystallisation
}

\author{
Stefanie E. Glassford ${ }^{a}$, Lata Govada ${ }^{b}$, Naomi E. Chayen ${ }^{b}$ Bernadette Byrne $^{c}$, \\ Sergei G. Kazarian ${ }^{\mathrm{a}, *}$
}

${ }^{a}$ Department of Chemical Engineering, Imperial College London, SW7 2AZ, U.K.

${ }^{\mathrm{b}}$ Department of Surgery and Cancer, Imperial College London, SW7 2AZ, U.K.

${ }^{c}$ Department of Molecular Bioscience, Imperial College London, SW7 2AZ, U.K.

*Corresponding author. E-mail address: s.kazarian@imperial.ac.uk (S.G. Kazarian)

\begin{abstract}
Protein crystallisation is of great importance within structural proteomic projects where considerable time and effort is spent trying to obtain high resolution structural information from the X-ray diffraction of protein crystals. The crystallisation process is largely empirical due to a lack of understanding of crystallisation kinetics and it is often very challenging to obtain crystals of sufficient size and quality for X-ray analysis and data collection. This paper presents the use of micro Attenuated Total Reflection (ATR) - Fourier Transform Infrared (FTIR) spectroscopic imaging as an analytical method for the measurement of the growth of protein crystals using the hanging drop approach. As well as allowing for the measurement of in-situ growth of protein crystals, the high spatial resolution of this method allows the detection of micro-crystals. The use of this technique could be very advantageous for protein structural studies since it allows both detection of the growth of small protein crystals and provides chemical information as the crystals form.
\end{abstract}

Keywords: Total internal reflection; FT-IR spectroscopy; Protein crystals; Spatial resolution; ATR imaging

\section{Introduction}

The crystallisation of proteins in order to determine their 3D structure has become an increasingly challenging area of research, especially with the continuing development of structural proteomics projects. A lot of time and effort is spent both generating the target protein and in submitting the protein to both preliminary and optimisation crystallisation trials with the aim of gaining their 3D structure from X-ray crystallography, the best available method for obtaining high resolution structural information [1]. However, despite many efforts focussing on rationalisation, the 
crystallisation of proteins is still largely an empirical process and involves a significant amount of trial and error [2]. Even when protein crystals are formed, it is still very difficult to obtain ones that are useful for X-Ray diffraction [2]. Often protein crystals can be too small or slightly imperfect. In addition to this, there is currently no easy way to distinguish between a protein and salt crystal within the crystallisation drop meaning that time and resources are often wasted optimising conditions, only to discover during final stages that the crystal is in fact not proteinaceous [3]. There are currently several crystallisation methods used in structural biology laboratories. The traditional approaches employing vapour diffusion methods such as hanging or sitting drop are the most widely used, however other methods including microbatch have been successfully used to crystallise a number of proteins. Other novel methods, including the use of porous surfaces and seeding technologies [4] have been developed along with significant progress in nanocrystallography [5] as well as specialized techniques such as lipidic cubic phase [6]and bicelle crystallisation [7] suitable for membrane proteins. Despite these exciting developments, hanging drop or sitting drop are still the most commonly employed methods. Hanging and sitting drop crystallisation are vapour diffusion methods, where a small drop of protein and precipitant solution is equilibrated against a reservoir containing a higher concentration of precipitant. As the drop equilibrates against the reservoir, the concentration of protein within the drop increases to the level required for the nucleation and growth of crystals [8].

As mentioned above, the optimum crystallisation conditions for each protein are identified by extensive screening. The development of new analytical processes to aid in the understanding of the crystallisation kinetics by measuring their growth in situ would greatly facilitate the development of rational crystallisation approaches. This paper introduces the use of Micro ATR FTIR spectroscopic imaging as a method that can be used to study the in-situ growth of protein crystals in a hanging drop configuration. The chemical specificity of infrared spectroscopy means that crystals can easily be identified as protein and the high spatial resolution can allow for the detection of micro crystals. The technique could also provide some insight into structural kinetics of crystallisation through the use of secondary structure analysis of the protein molecules.

Infrared spectroscopy has already been extensively used for the study of protein behaviours such as surface adsorption [9] and in solution [10]. Characteristic absorption bands for proteins are sensitive to protein conformation and there are many examples in literature for the assignment of protein secondary structure based on the analysis of Amide I band [11, 12, 13]. Chemical imaging using infrared spectroscopy offers the opportunity to study protein crystallisation in situ. Macro ATR FTIR spectroscopic imaging applied to the study of protein crystallisation in a high throughput manner has previously been shown by Chan et al. [3]. This work demonstrated the applicability of ATR FTIR 
spectroscopic imaging to distinguish between multiple crystallisation conditions simultaneously using a macro approach, where the spatial resolution is limited to $40 \mu \mathrm{m}$ using a zinc selenide ATR accessory or 15-20 $\mu \mathrm{m}$ with a diamond accessory [3]. Macro ATR FTIR imaging has also been used for the study of the effect of different surface properties on protein adsorption and crystallisation by preparing a gradient monolayer on the surface of a silicon ATR accessory [14]. However, the spatial resolution in that study was limited to approximately $84 \mu \mathrm{m}$ [14]. It would, therefore be useful to obtain chemical images of protein crystals with a higher spatial resolution to allow the detection of micro crystals as well as attempt to measure protein crystal growth in-situ.

ATR FTIR spectroscopic imaging is an extremely versatile analytical technique that allows for the measurement of chemical specific images of a sample, without the need for additional dyes. An FTIR spectrometer equipped with an accessory that contains a single bounce internal reflection element (IRE) coupled with an FPA detector enables spatially resolved chemical information to be obtained from the sample by measuring $4000-16,000$ spectra simultaneously from different locations within the sample. The nature of the ATR approach is such that infrared light only interacts with the sample within a few micrometers from the surface of the internal reflection element. This limited probing depth means that it is particularly applicable to the study of aqueous samples but it does require good contact between a particular sample and the surface of the ATR element [15]. Micro ATR FTIR combines a Cassegrain type objective and an infrared microscope operating in reflection mode, shown in Fig. 1. The angle of incidence of this type of objective is ca. $30^{\circ}$ meaning a Germanium ATR element $(n=4)$ is used to ensure total internal reflection of the IR beam occurs. The high refractive index of the Germanium element combined with the IR microscope objective means that spatial resolution of approximately $4 \mu \mathrm{m}$ can be achieved [16]. This has enabled the study of many samples previously excluded from FTIR imaging studies due to inadequate spatial resolution. The field of view with this approach is limited and the size of the imaging area has been measured to be approximately $64 \mu \mathrm{m} \times 64 \mu \mathrm{m}$ [16]. Despite this small imaging area, Micro ATR FTIR spectroscopic imaging has great potential for the studies of polymeric materials $[17,18,19,20]$, cross-sections of paintings [21] and biomedical materials where it has already been employed to analyse cross sections of hair [22], breast cancer tissue [16] aorta and arteries [23] as well as to chemically image live cancer cells [15].

The Micro ATR FTIR set up is particularly amenable to the study of hanging drop protein crystallisation. The Ge ATR crystal is removable, as reported previously in reference 15 . As such a drop of protein solution and precipitant solution can be deposited directly onto the Ge crystal and a reservoir can then be placed underneath the drop in exactly the same manner as that which is applied in structural biology labs. Confocal Raman microscopy has also been applied to the in situ 
measurement of hanging drop protein crystallisation to monitor the change in concentration of protein within the crystallisation drop [24, 25]. Micro ATR FTIR imaging is an imaging technique and thus requires shorter measurement and sample preparation times to obtain the same number of spectra from the sample in comparison with Confocal Raman microscopy. Also, despite the small imaging area of Micro ATR FTIR, it is possible to measure the growth of multiple crystals using this approach as will be demonstrated below.

This novel application of Micro ATR FTIR opens up exciting opportunities for the dynamic study of protein crystallisation by measuring the growth of crystals in-situ. The high spatial resolution of the technique allows for the measurement of the early stages of crystal growth through the detection of micro crystals and imaging their growth. This could facilitate the identification of conditions optimal for crystal growth, quickly and easily without need to damage or move the crystal drop. It has potential to be further developed for the study of protein structure in proteins where it is very hard to obtain X-Ray standard crystals, through FTIR spectroscopic analysis of protein secondary structure. This method could also be applied to other areas of research where crystallisation is a key factor, for example pharmaceuticals.

This paper shows the micro ATR FTIR spectroscopic imaging of three different proteins. Lysozyme and Thaumatin are both easily crystallised and therefore good model proteins with which to develop a new technique. The third protein studied was Lobster $\alpha$-crustacyanin which has previously been studied to understand the natural blue colouration of raw lobster shells and is more challenging to crystallise [26].

\section{Experimental}

\subsection{Materials}

Three proteins Lysozyme, Thaumatin and $\alpha$-crustacyanin were studied, each with different crystallisation conditions at $20^{\circ} \mathrm{C}$.

Hen egg-white Lysozyme (L 7651), Thaumatin from Thaumatococcus danielii (T 7638) and all the chemicals used in the reservoir solutions were obtained from Sigma-Aldrich. Alpha crustacyanin was provided by Peter Zagalsky of Royal Holloway University of London.

\subsection{Crystallisation conditions}

A solution of $40 \mathrm{mg} / \mathrm{ml}$ Lysozyme in $0.1 \mathrm{M}$ Sodium Acetate buffer, $\mathrm{pH} 4.5$ was prepared. The corresponding reservoir solution contained $6 \% \mathrm{w} / \mathrm{v} \mathrm{NaCl}$ in $0.1 \mathrm{M}$ Sodium Acetate buffer, $\mathrm{pH}$ 4.5. Thaumatin solution at $30 \mathrm{mg} / \mathrm{ml}$ in $0.8 \mathrm{M}$ Sodium Potassium Tartrate, $\mathrm{pH} 6.8$ was crystallised using a reservoir solution containing 0.1M BIS-TRIS Propane (BTP) in the same buffer. $\alpha$-crustacyanin 
solution at $5 \mathrm{mg} / \mathrm{ml}$ in $28 \% \mathrm{w} / \mathrm{v}$ Polyethylene glycol monomethyl ether 2,000 was equilibrated against a reservoir solution of $0.1 \mathrm{M} \mathrm{BIS-TRIS} \mathrm{pH} \mathrm{6.5.}$

All crystallisation drops were deposited on the Ge ATR element and equilibrated against $200 \mu \mathrm{L}$ of their reservoir solutions. $2 \mu \mathrm{L}$ drops each of Lysozyme and Thaumatin were set up in a 1:1 protein to reservoir ratio while alpha crustacyanin was set up in a 2:1 ratio.

\subsection{Micro ATR FTIR spectroscopic imaging measurements}

All ATR-FTIR imaging measurements were carried out on a continuous scan spectrometer (Varian) coupled to an IR microscope equipped with a liquid nitrogen cooled focal plane array detector (FPA). A removable Germanium ATR crystal (Refractive index, $n=4$ ) was attached to a Cassegrain objective mounted on the IR microscope. The $64 \times 64$ pixels FPA detector has a measured imaging area of 64 $\mu \mathrm{m} \times 64 \mu \mathrm{m}$. Each measurement consists of 250 scans co-added at $8 \mathrm{~cm}^{-1}$ spectral resolution and have been ratioed against a background spectrum which was measured prior to protein deposition for each new sample. The time taken for each measurement was 7 minutes.

The crystallisation drop was deposited directly onto the measuring surface of the Germanium ATR element which was then mounted onto the IR microscope objective. A small reservoir of solution is placed beneath the crystallisation drop and the system sealed using silicon grease, in a similar manner to commercially available Hanging drop crystallisation plates (Corning Life Sciences). This set up is shown in Fig. 1.

\subsection{Optical Images}

Optical images for Lysozyme crystals were obtained using the 40x objective on the IR microscope. In the case of Thaumatin and Lobster $\alpha$-crustacyanin, optical images were obtained using a Leica optical microscope.

\section{Results and discussion}

\subsection{Lysozyme}

Lysozyme was used as a model protein for the development of the micro ATR-FTIR method since it is readily crystallisable. ATR FTIR imaging measurements were taken as soon as the trial was set up and at subsequent forty minute intervals. Over time the growth of a Lysozyme crystal could be seen in the bottom left corner of the image, shown in Fig. 2. Spectra extracted from the centre region of this protein crystal (Fig. 2) show that the growth of the protein crystal is marked by the appearance of the characteristic Amide I and II bands. The ATR FTIR images were generated by plotting the distribution of absorbance of the Amide II band at $1540 \mathrm{~cm}^{-1}$ within the imaging area. From the ATR 
FTIR images, the maximum size of the crystal measured can be approximated to $29 \mu \mathrm{m}$, however it is clear than this is only a part of a crystal as the crystal had partly grown outside the field of view.

Visible images of the measuring surface of the Germanium crystal were taken after 20 hours, shown in Fig. 3. Since optical images require the removal of the objective from the IR microscope, only one image was taken during the whole trial in order to minimise disturbance to the crystallisation drop. Moving the Germanium crystal can also affect the ratio with background spectra and hence should be avoided as much as possible. Water vapour can be an issue with IR spectroscopy and removing the objective exposes the spectrometer to the local environment through the gap in equipment where the objective would be. This increases the amount of water vapour within the spectrometer which interferes with the infrared light path, affecting the quality of spectral measurements increase. Therefore, to minimise the effect of this and allow the system to return to equilibrium, a time interval of 30 minutes was observed before taking a measurement after each time the ATR element was removed.

From the visible image, the presence of multiple Lysozyme crystals can be seen, with an approximate size of $50 \mu \mathrm{m}$, indicating that half of one of these crystals was captured using micro ATR FTIR imaging. This highlights the main limitation of this method; the limited imaging area. Therefore, for proteins that grow rapidly to form big crystals, it may not always be possible to capture their growth using micro ATR FTIR imaging. There is no guarantee that a nucleation site will occur within the imaging area and hence there is still a degree of trial and error within this approach. The exact location of the imaging area on the germanium crystal is also not known precisely, the black dashed box in the visible image shows an estimated position (from the shape and size of the crystal on ATR image). Opportunities exist in which this could be determined in order to overcome this limitation. If the exact location of the ATR imaging area was known then it may be possible to ensure crystal growth in this area through the use of surface modifications or seeding technologies, for example.

\subsection{Thaumatin}

Thaumatin is another protein that is well characterised and crystallises readily, although over a longer time than Lysozyme. ATR FTIR imaging measurements were taken at time $=0,24 \mathrm{~h}$ and $28 \mathrm{~h}$ (Fig. 4) where the ATR images were generated by plotting the distribution of the Amide II band at $1525 \mathrm{~cm}^{-1}$ within the imaged area. Spectra extracted from the highlighted region are also shown and a visible image Thaumatin crystals on the germanium surface. After 24 hours several small crystals have grown within the ATR imaging area, varying between $6-15 \mu \mathrm{m}$ in size. Then at $28 \mathrm{~h}$, the size of these crystals has reduced to a maximum of $6 \mu \mathrm{m}$. This would suggest that as the crystals increase in 
size, the effect of gravity means that the area of crystal in contact with the Ge surface decreases. Again an optical image was taken at the latest stage of the trial to minimise disturbance to the sample. The image shows many protein crystals across the Ge surface; however from the image it is hard to distinguish individual crystals.

Measurements of Thaumatin were repeated and ATR FTIR images are shown in Fig. 5 along with spectra extracted from the highlighted region. The spectra appear to be noisier than the previous measurements due to the presence of a larger amount of water vapour in the system. As before, after $24 \mathrm{~h}$ several protein crystals can be observed in the ATR FTIR image with a maximum size of approximately $15 \mu \mathrm{m}$ then after $48 \mathrm{~h}$ the number and size of Thaumatin crystals measured has reduced; the biggest one approximately $6 \mu \mathrm{m}$. This confirms that as time increases and the crystals become sufficiently large, they are too heavy and less surface area is in contact with the ATR element. A visible image (Fig. 5) of the germanium surface at $48 \mathrm{~h}$ shows Thaumatin crystals of approximately $20 \mu \mathrm{m}$. These measurements of Thaumatin crystallisation show that micro ATR can provide information during the initial growth of the crystals meaning that it is a technique suitable for the early stages of crystallisation. Further analysis of the protein spectra obtained during this early, critical stage in the process could yield information about crystallisation kinetics. Additionally, this analysis has the advantage of differentiation between salt and protein crystals than is possible using other techniques.

\subsection{Lobster alpha crustacyanin}

To determine if Micro ATR imaging could be applied to more challenging proteins, the hanging drop crystallisation of lobster alpha crustacyanin was studied. Crystallisation drops of lobster alpha crustacyanin normally result in the formation of a large number of small blue crystals which in some cases may be too small for use in X-Ray studies.

$\alpha$-Crustacyanin is known to take approximately one day to crystallise under standard laboratory conditions. ATR FTIR imaging measurements were taken at $0,48 \mathrm{~h}$ and $96 \mathrm{~h}$, with ATR images, generated from plotting the distribution of Amide II band at $1540 \mathrm{~cm}^{-1}$, shown in Fig. 6 along with spectra extracted from the highlighted area. After $48 \mathrm{~h}$, a large amount of the protein has aggregated onto the surface of the Ge element although several micro-crystals can also be detected, shown by the small regions of higher concentration of protein. A further measurement at $96 \mathrm{~h}$ shows several large regions of high concentration of proteins. From the visible image (Fig. 6) it can be seen 
that the crystals did not grow bigger than $10 \mu \mathrm{m}$, therefore these regions are most likely several crystals that have agglomerated together (largest region from ATR images $\sim 15 \mu \mathrm{m}$ ). It should be noted that the optical image obtained shows a larger area of the Ge element surface than the imaging area using ATR FTI therefore a direct comparison of crystals is not possible. Measurements were repeated for a new crystallisation drop, at time $1 \mathrm{~h}, 21 \mathrm{~h}$ and $48 \mathrm{~h}$. ATR images and spectra from highlighted region are shown in Fig. 7 along with an optical image of the protein crystals on the Ge surface. These correlate with the previous trial; by $48 \mathrm{~h}$ a large amount of protein had aggregated on the surface with several micro crystals present.

The measurement of the crystallisation of $\alpha$-crustacyanin by this approach demonstrates the applicability to proteins that are more challenging to crystallise and highlights the ability to detect the formation of micro crystals. Previously in macro ATR imaging studies, the spatial resolution was such that protein crystals had to be much more significant in size before they could be detected. This means it could be applied to the study of proteins that do not form crystals big enough or of high enough quality for X-Ray diffraction studies as well as to detect the early stages of crystal growth.

\section{Conclusions}

This paper demonstrates the novel application of micro ATR FTIR spectroscopic imaging to the study of hanging drop protein crystallisation. The deposition of a protein solution directly onto a Germanium ATR objective means that the growth of protein crystals can be measured in-situ without the need for additional measures to identify them as actual protein crystals, during the initial stages of crystal growth. The measurement of multiple protein crystals simultaneously has also been shown to be possible. The high spatial resolution of Micro ATR FTIR images has allowed the detection of protein crystals approximately $6 \mu \mathrm{m}$ in size. The high spatial measurement of crystal growth in-situ could offer exciting opportunities for the study of target proteins, especially those which do not produce crystals of the size required for X-Ray crystallography. It could also have potential application within other areas of research where the understanding of crystallisation is important such as drug design and development in pharmaceuticals.

\section{Acknowledgments}

S.E.G. is funded by a BBSRC Targeted Priority Studentship in the area of Bioprocessing. S.G.K. acknowledges the research funding from the European Research Council under the European Community's Seventh Framework Programme (FP7/2007-2013)/ERC advanced grant agreement no. [227950]. N.E. C. acknowledges funding from the UK Engineering and Physical Sciences Research 
Council EP/G027005/1. We thank Peter Zagalsky of Royal Holloway University of London for supplying Alpha crustacyanin. 


\section{References}

[1] A. McPherson, Methods 34 (2004) 254-265.

[2] N.E. Chayen, Current Opinion in Structural Biology 14 (2004) 577-583.

[3] K.L.A. Chan, L. Govada, R.M. Bill, N.E. Chayen, S.G. Kazarian, Analytical Chemistry 81 (2009) 37693775.

[4] V. Bolanos Garcia, N. Chayen, Progress in Biophysics and Molecular Biology 101 (2009) 3-12.

[5] E.R. Bodenstaff, F.J. Hoedemaeker, M.E. Kuil, H.P.M. de Vrind, J.P. Abrahams, Acta Crystallographica Section D-Biological Crystallography 58 (2002) 1901-1906.

[6] V. Cherezov, Current Opinion in Structural Biology 21 (2011) 559-566.

[7] S. Faham, J.U. Bowie, Journal of Molecular Biology 316 (2002) 1-6.

[8] N.E. Chayen, Acta Crystallographica Section D-Biological Crystallography 54 (1998) 8-15.

[9] A. Sethuraman, G. Belfort, Biophysical Journal 88 (2005) 1322-1333.

[10] K.A. Oberg, A.L. Fink, Analytical Biochemistry 256 (1998) 92-106.

[11] W.K. Surewicz, H.H. Mantsch, D. Chapman, Biochemistry 32 (1993) 389-394.

[12] P.I. Haris, F. Severcan, Journal of Molecular Catalysis B-Enzymatic 7 (1999) 207-221.

[13] A. Barth, C. Zscherp, Quarterly Reviews of Biophysics 35 (2002) 369-430.

[14] S. Glassford, K.L.A. Chan, B. Byrne, S.G. Kazarian, Langmuir 28 (2012) 3174-3179.

[15] M.K. Kuimova, K.L.A. Chan, S.G. Kazarian, Applied Spectroscopy 63 (2009) 164-171.

[16] S.G. Kazarian, K.L.A. Chan, Applied Spectroscopy 64 (2010) 135A-152A.

[17] A. Gupper, P. Wilhelm, M. Schmied, S.G. Kazarian, K.L.A. Chan, J. Reussner, Applied Spectroscopy 56 (2002) 1515-1523.

[18] A. Gupper, P. Wilhelm, G. Kothleitner, K.J. Eichhorn, G. Pompe, Macromolecular Symposia 205 (2004) 171-180.

[19] D.J. Nagle, G.A. George, L. Rintoul, P.M. Fredericks, Vibrational Spectroscopy 53 (2010) 24-27.

[20] G.C. Eder, L. Spoljaric-Lukacic, B.S. Chernev, Analytical and Bioanalytical Chemistry 403 (2012) 683-695.

[21] M. Spring, C. Ricci, D.A. Peggie, S.G. Kazarian, Analytical and Bioanalytical Chemistry 392 (2008) 37-45.

[22] K.L.A. Chan, S.G. Kazarian, A. Mavraki A., D. R. Williams Applied Spectroscopy 59 (2005) 149155.

[23] F. Palombo, S.G. Cremers, P.D. Weinberg, S.G. Kazarian, Journal of the Royal Society Interface 6 (2009) 669-680.

[24] K. Noda, H. Sato, S. Watanabe, S. Yokoyama, H. Tashiro, Applied Spectroscopy 61 (2007) 11-18.

[25] J. Vongsvivut, S. Watanabe, K. Noda, H. Sato, H. Tashiro, Scienceasia 34 (2008) 400-408. 
[26] N.E. Chayen, M. Cianci, J.G. Grossmann, J. Habash, J.R. Helliwell, G.A. Nneji, J. Raftery, P.J.

Rizkallah, P.F. Zagalsky, Acta Crystallographica Section D-Biological Crystallography 59 (2003) 2072-2082. 


\section{Figure captions:}

Fig. 1. Schematic of Micro ATR imaging optics with protein crystallisation experiment.

Fig. 2. Micro ATR images of Lysozyme crystallisation and spectra extracted from region marked by box. $A=0 \mathrm{~min}$ (spectra only), $B=40 \mathrm{~min}$ (spectra only), $C=80 \mathrm{~min}, \mathrm{D}=110 \mathrm{~min}, \mathrm{E}=140 \mathrm{~min}, \mathrm{~F}=20$ h. The ATR images show distribution of absorbance of the Amide II band at $1540 \mathrm{~cm}^{-1}$. Spectra A and B show absorption of the crystallisation solution prior to formation of protein crystals, therefore the main band at ca. $1640 \mathrm{~cm}^{-1}$ is from the bending mode of water.

Fig. 3. Visible image of Lysozyme crystals on surface of Ge ATR element. Dashed box shows approximate size of imaging area.

Fig. 4. i) Micro ATR images of Thaumatin crystals. ii) Extracted spectra from region marked by box on the image: $A=0 \mathrm{~h}$ (spectra only), $\mathrm{B}=24 \mathrm{~h}$ (crystal size 6-15 $\mu \mathrm{m}$ ), $\mathrm{C}=28 \mathrm{~h}$ (crystal size $<6 \mu \mathrm{m}$ ). The ATR images show distribution of absorbance of the Amide II band at $1525 \mathrm{~cm}^{-1}$. Spectrum A shows absorption prior to formation of protein crystals. iii) Visible image of Thaumatin crystals on surface of Ge ATR element.

Fig. 5. i) Micro ATR images of Thaumatin crystals. ii) Extracted spectra from area marked by box on image: $\mathrm{A}=24 \mathrm{~h}$ (crystal size $\sim 15 \mu \mathrm{m}$ ), $\mathrm{B}=48 \mathrm{~h}$ (crystal size $\sim 10 \mu \mathrm{m}$ ) The ATR images show distribution of absorbance of the Amide II band at ca. $1525 \mathrm{~cm}^{-1}$. iii) Visible image of Thaumatin crystals on surface of Ge ATR element.

Fig. 6. i) Micro ATR images of Lobster alpha crustacyanin crystallisation. ii) Extracted spectra from boxed area. $A=0 \mathrm{~h}$ (spectra only), B= 48 h (Crystal size $\sim 6 \mu \mathrm{m}), C=96 \mathrm{~h}$ (Crystal size $\sim 15 \mu \mathrm{m})$ The ATR images show distribution of absorbance of the Amide II band at ca. $1540 \mathrm{~cm}^{-1}$. Spectrum A shows absorption prior to formation of protein crystals. iii) Visible image of Lobster alpha crustacyanin crystals on surface of Ge ATR element.

Fig. 7. i) Micro ATR images of Lobster alpha crustacyanin crystallisation. ii) Visible image of Lobster alpha crustacyanin crystals on surface of Ge ATR element. iii) Extracted spectra from boxed area. $A=$ $1 \mathrm{~h}, \mathrm{~B}=21 \mathrm{~h}, \mathrm{C}=48 \mathrm{~h}$. The ATR images show distribution of absorbance of the Amide II band at ca. $1540 \mathrm{~cm}^{-1}$. Spectrum A shows absorption prior to formation of protein crystals. 


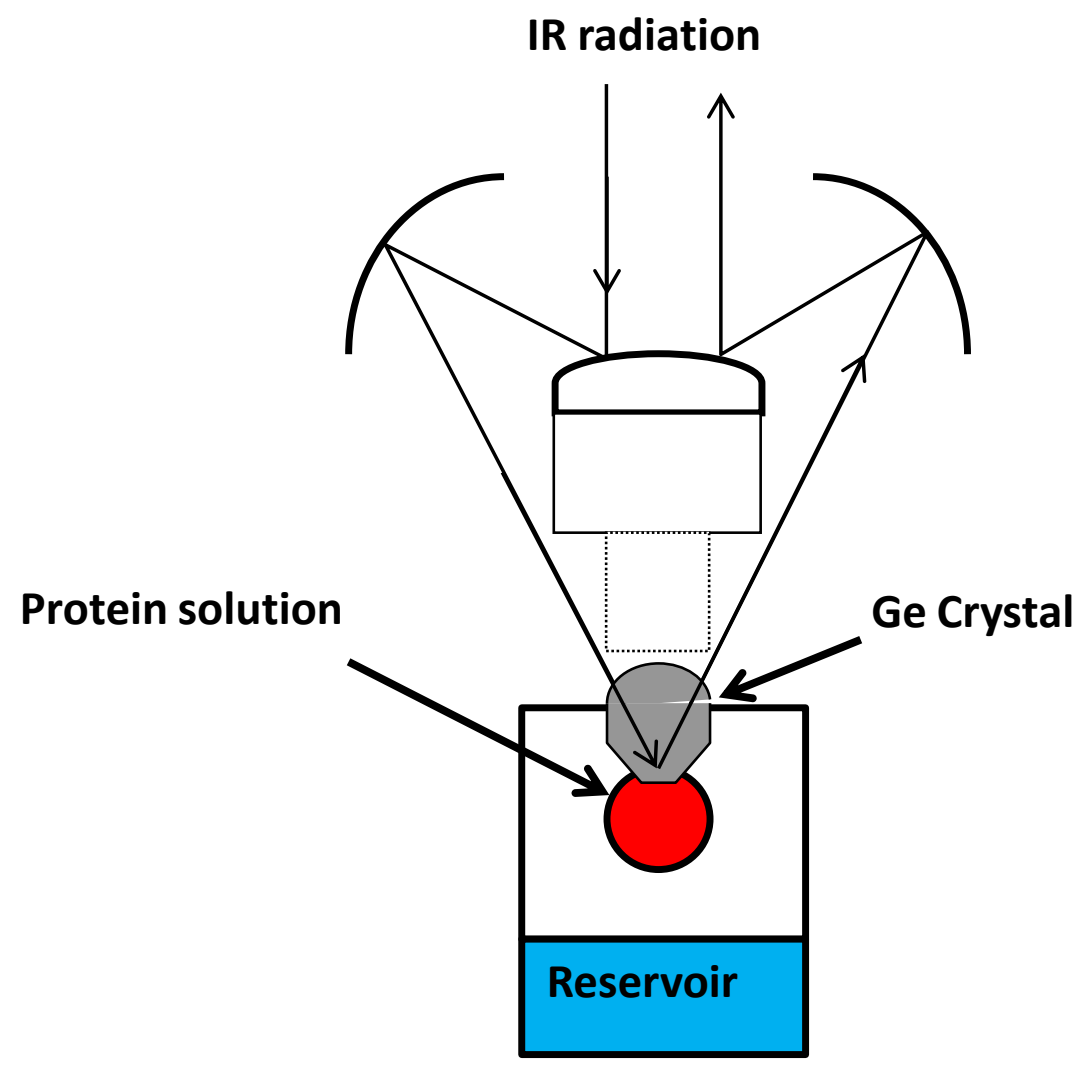




$$
\text { i }
$$
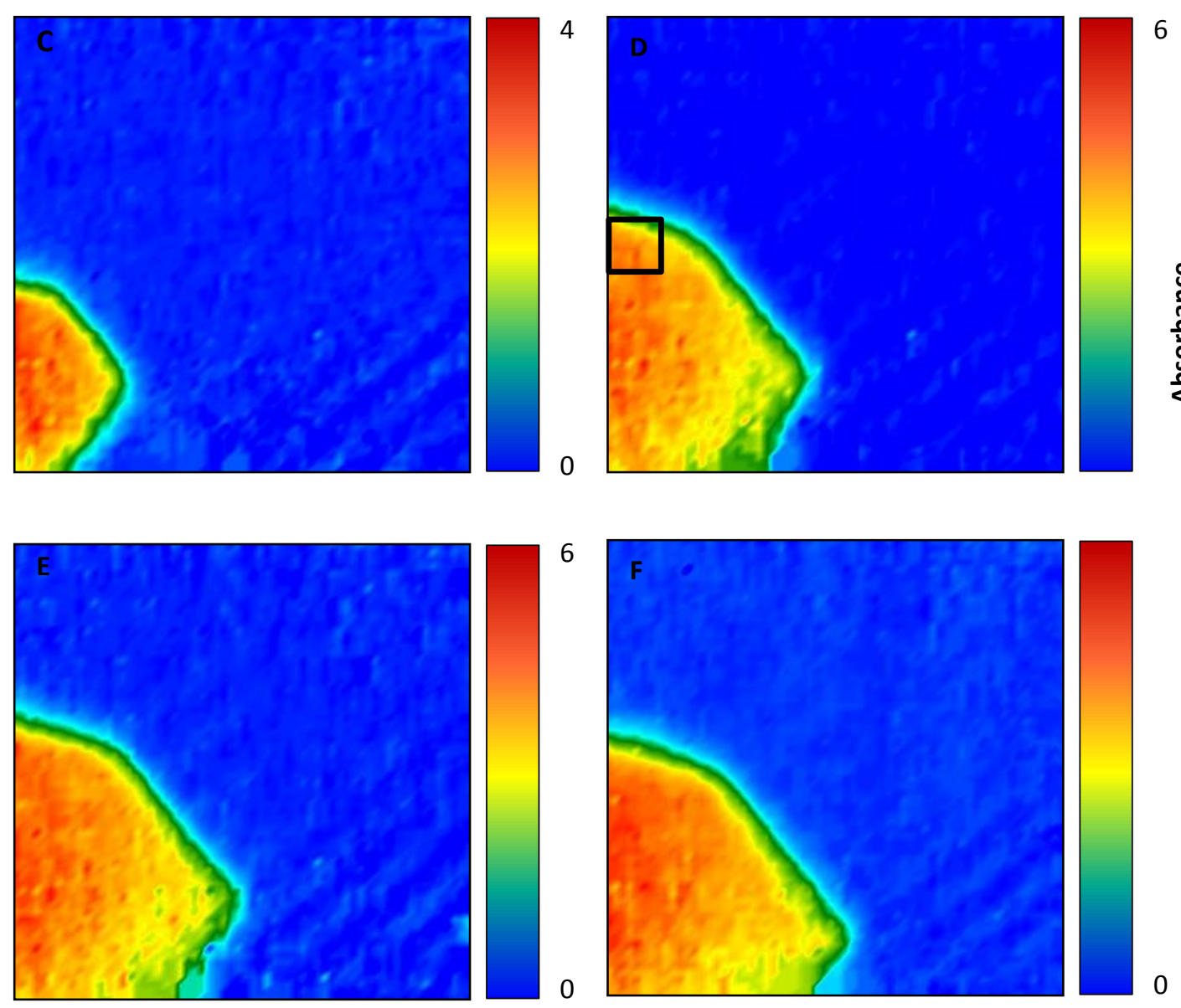

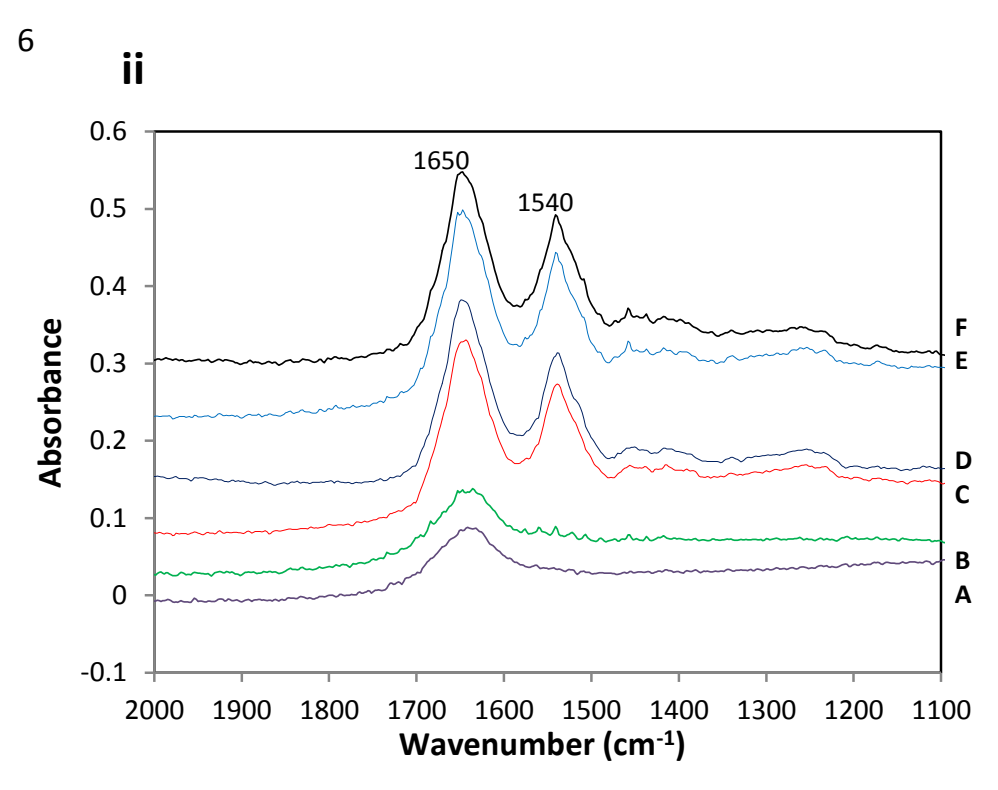


Figure 3

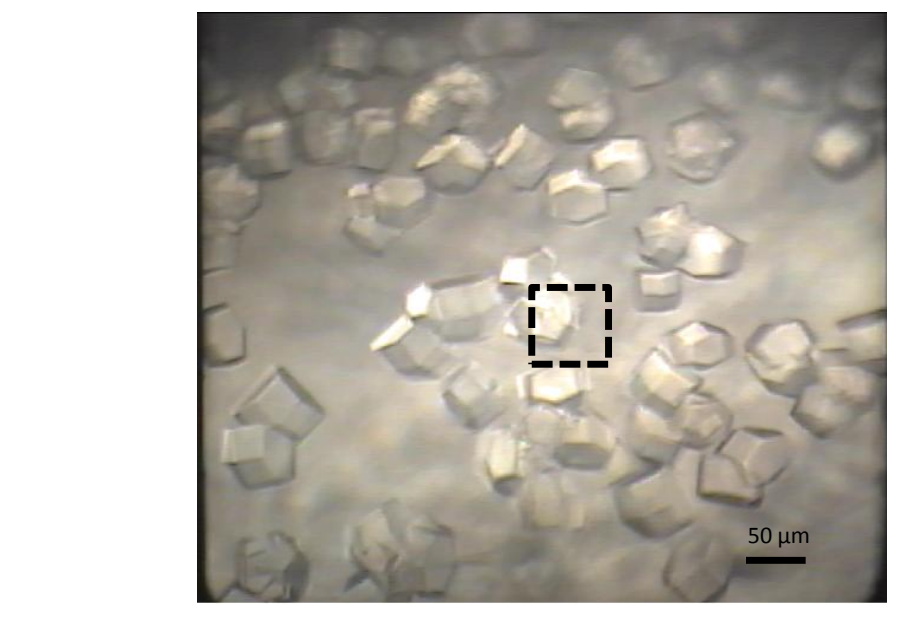


Figure 4

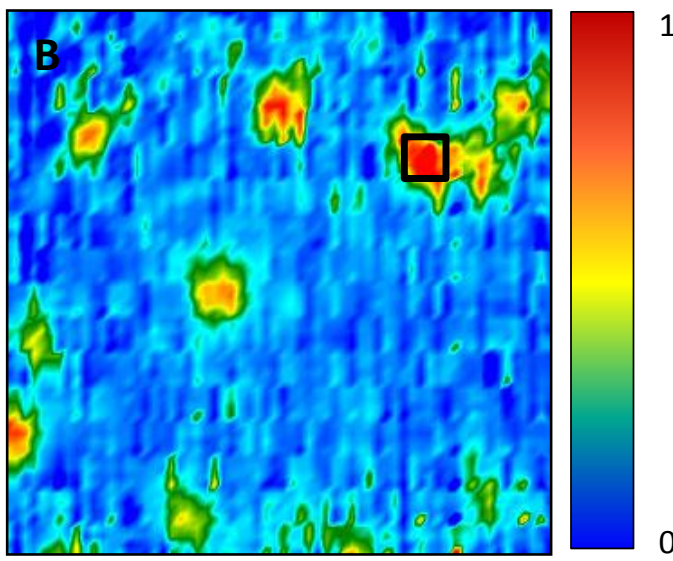

1

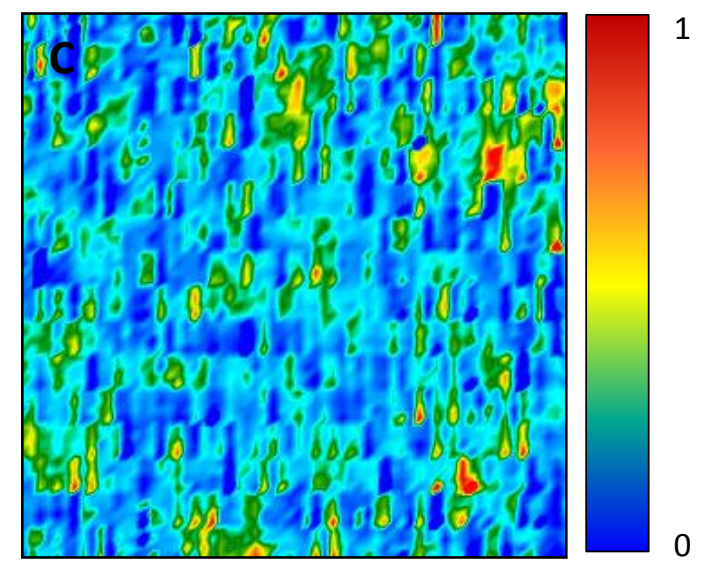

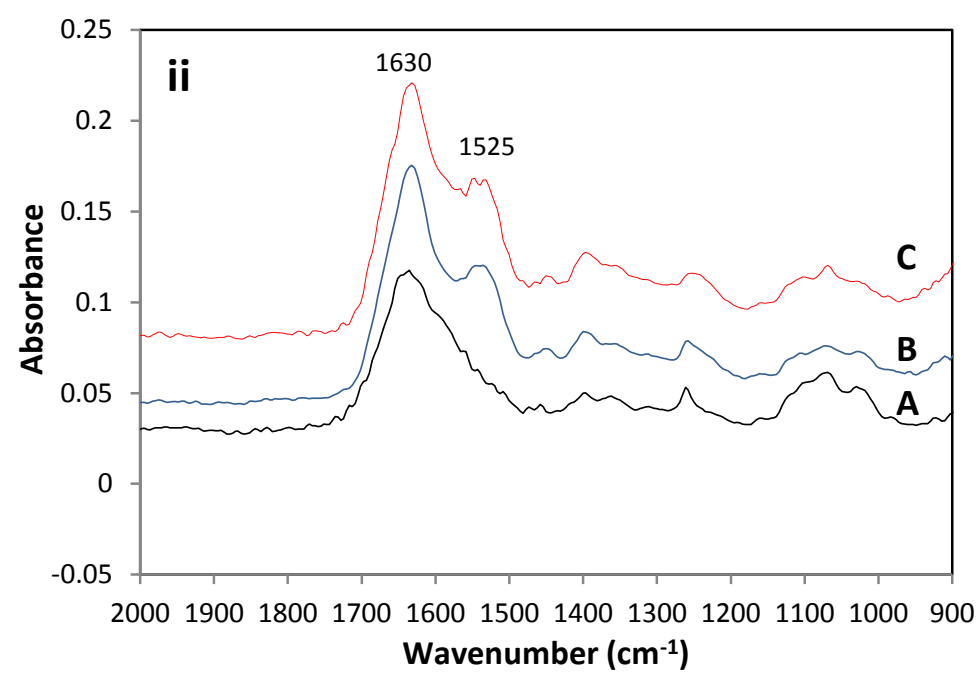

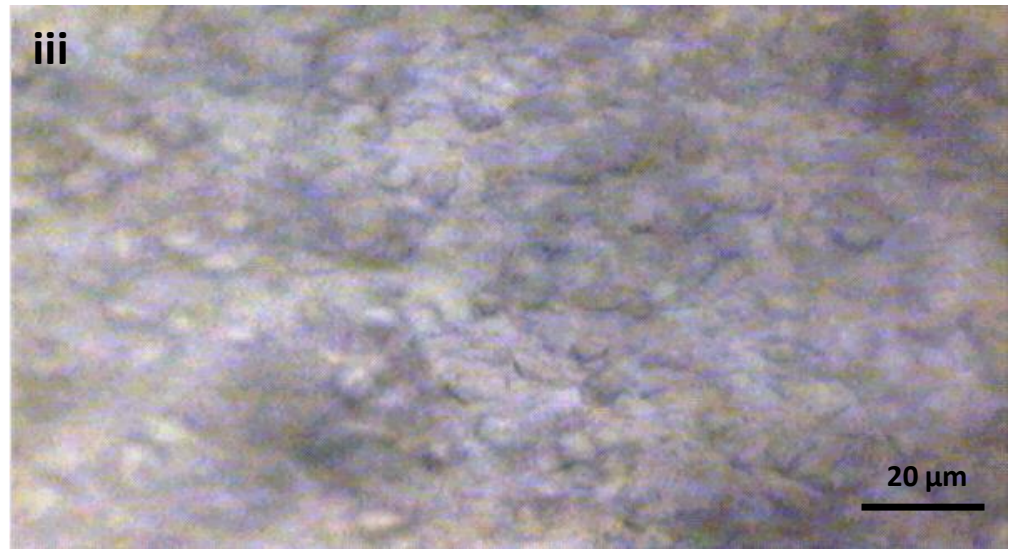
Wavenumber $\left(\mathrm{cm}^{-1}\right)$ 


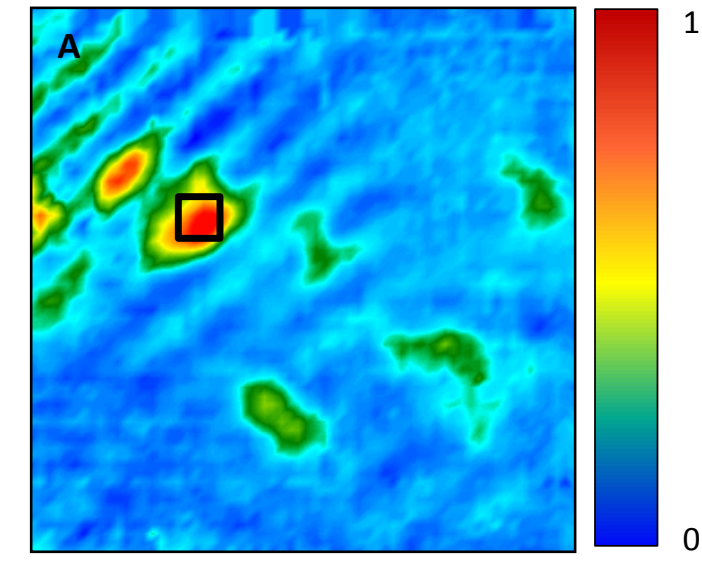

1.5
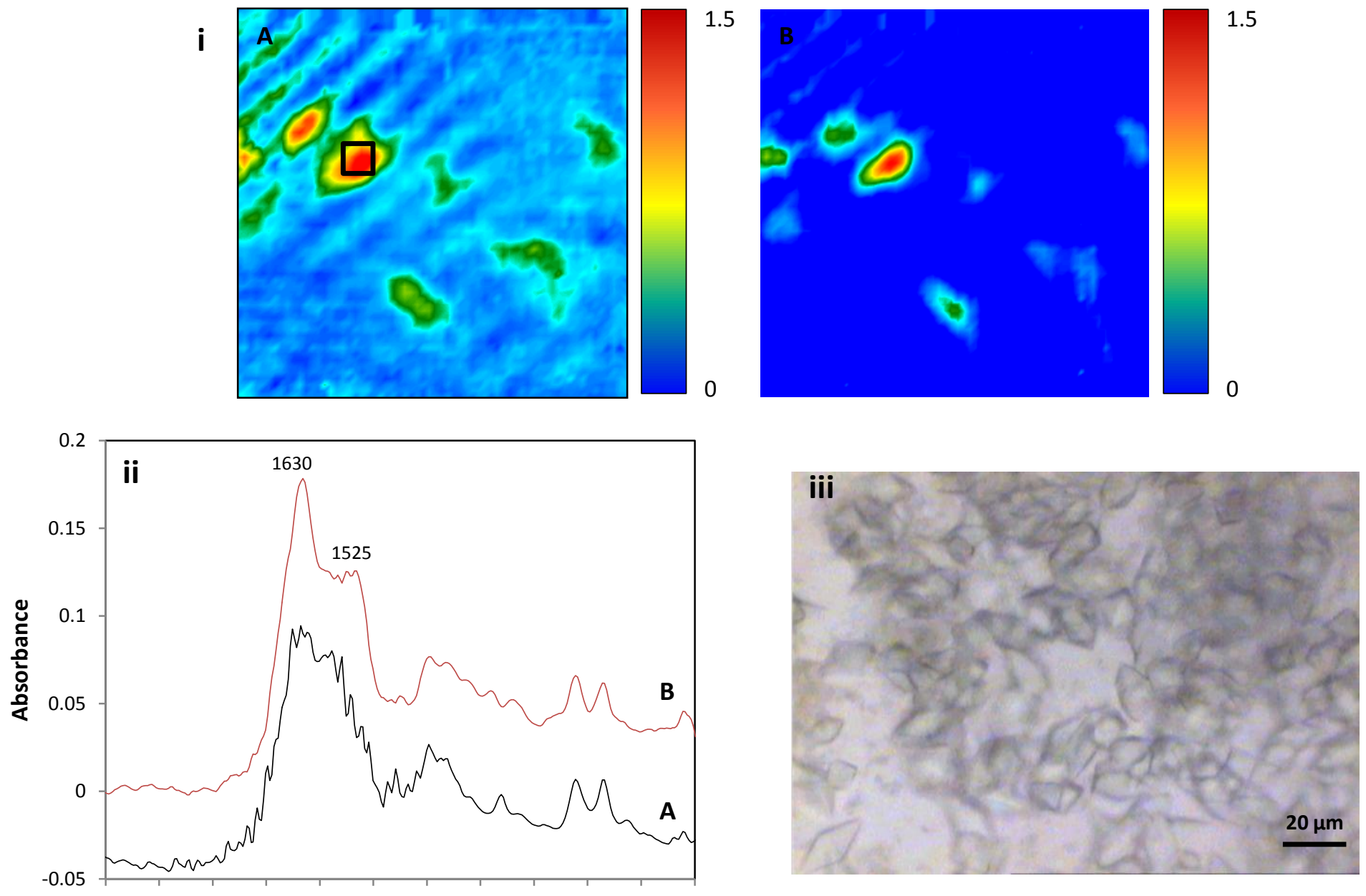

20001900180017001600150014001300120011001000900 Wavenumber $\left(\mathrm{cm}^{-1}\right)$ 

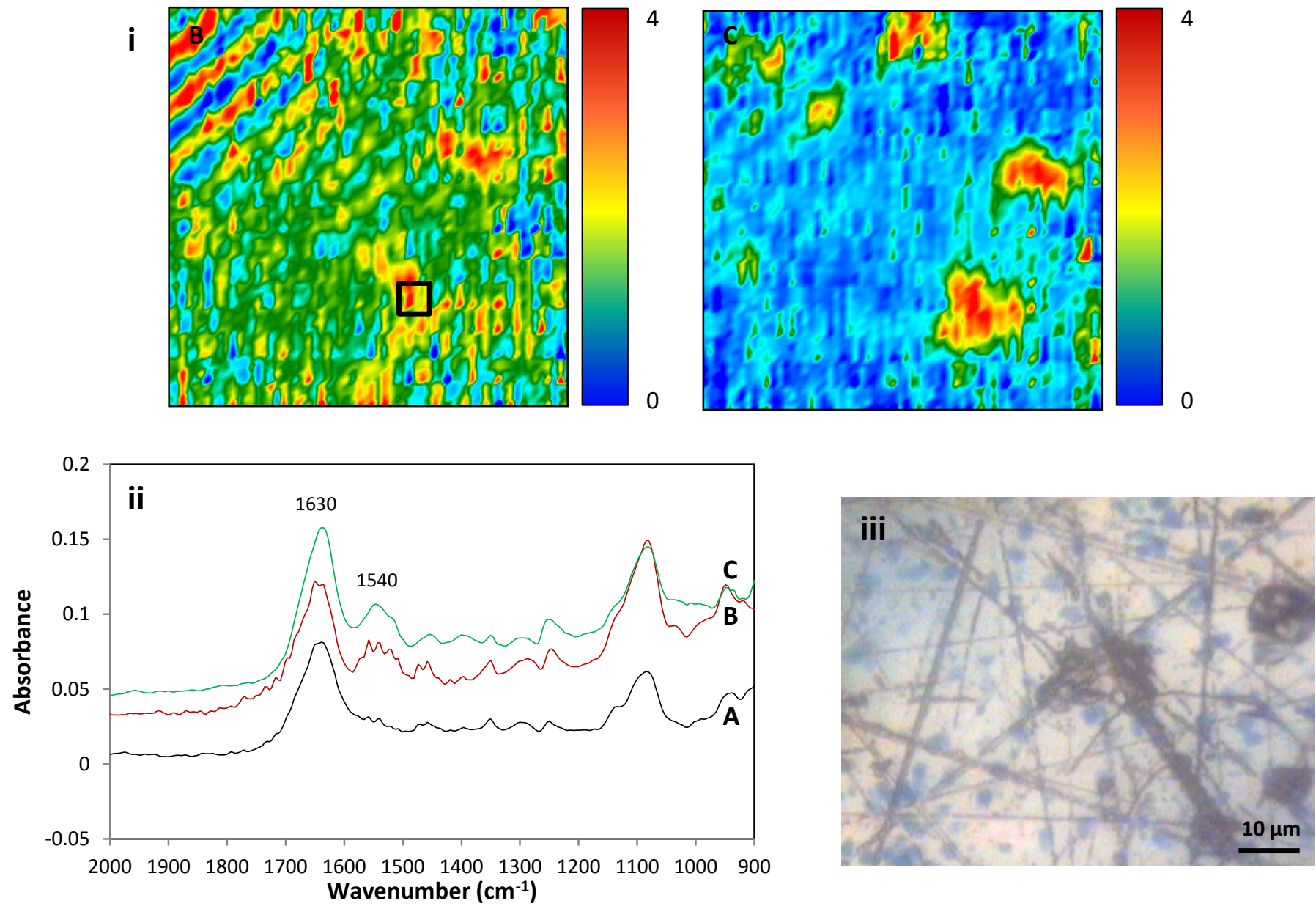

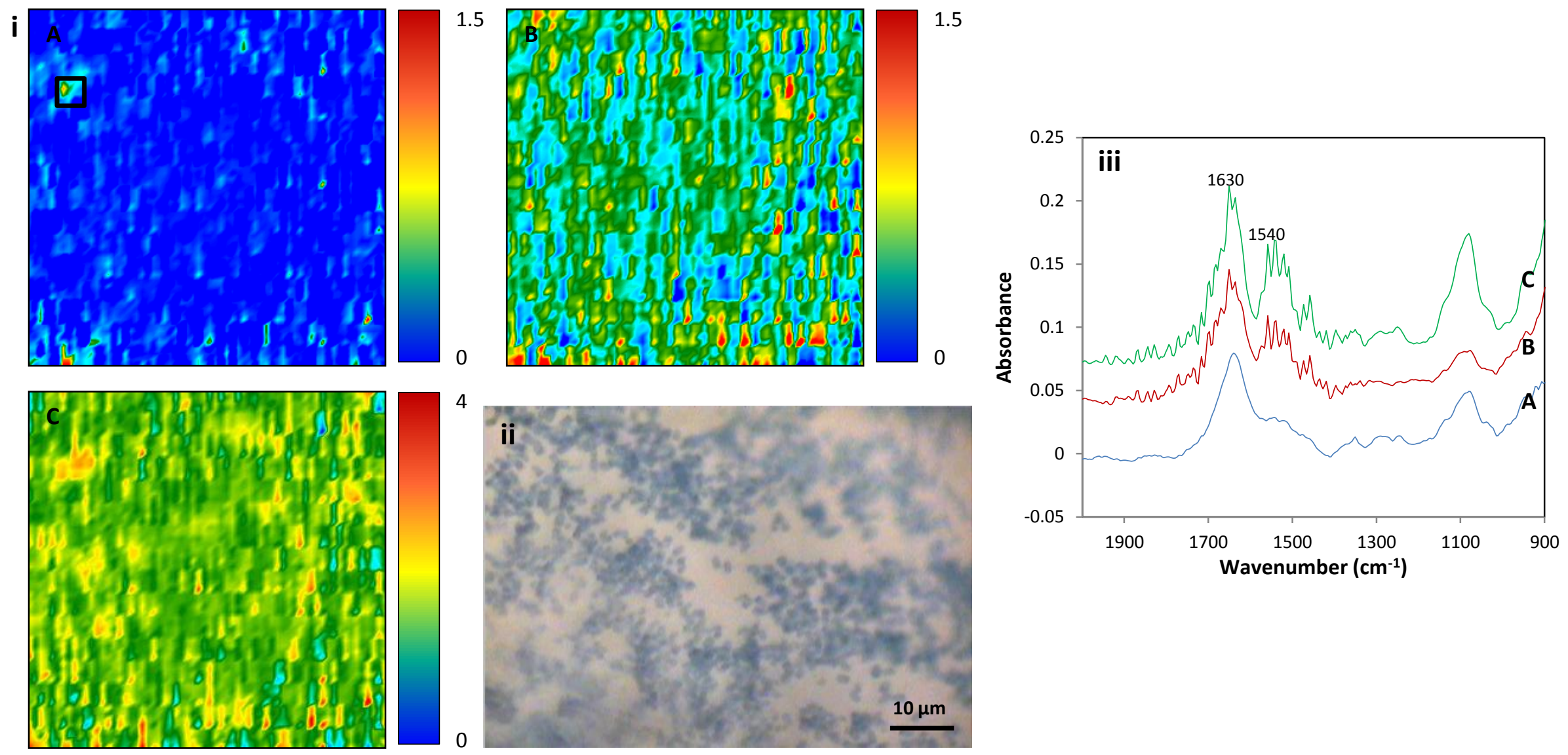